\title{
Immunosenescence of CD4+ T cells in male homosexual patients with HIV-1 infection
}

\author{
Li Li ${ }^{1}$, Linghang Wang ${ }^{1}$, Fengting $\mathrm{Yu}^{1}$, Siyuan Yang ${ }^{1}$, Shujing Song ${ }^{1}$, Yunxia Tang ${ }^{1}$, \\ Chengjie $\mathrm{Ma}^{1}$, and Xingwang $\mathrm{Li}^{1}$ \\ ${ }^{1}$ Capital Medical University Affiliated Beijing Ditan Hospital
}

April 28, 2020

\begin{abstract}
The average lifespan of HIV-infected subjects remains shorter compared to uninfected individuals. Immunosenescence may be responsible for this difference despite effective antiretroviral therapy (ART) with successful viral suppression. Here, we evaluated the effects of HIV and ART exposure on $\mathrm{T}$ cell aging in male homosexual HIV subjects. CD4+ T cell activation (HLA-DR+) and senescence (CD57+) markers were analyzed by flow cytometry, and telomere length was quantified by realtime PCR. Specifically, we observed an increase in activation and senescence markers on total CD4+ T cell populations in HIV-infected subjects. We also observed a reduction in senescence markers on terminally differentiated memory T(TemRA) cells and activation markers on central memory T(TCM), effector memory T(TEM), and TemRA cells in ART-treated HIV subjects. Furthermore, we also observed an extension of telomere length in memory CD4+ T cells, rather than naive CD4+ T cells, after viral control by ART. Our results indicate that HIV-infected patients exhibit a premature T cell aging phenotype with accelerated immune senescence. Partial recovery of immune senescence and differentiation aberrances is achieved in CD4+ $\mathrm{T}$ cells in HIV patients on ART. Overall, these results suggest that HIV infection, rather than ART exposure, influences the T cell aging process.
\end{abstract}

\section{Immunosenescence of $\mathrm{CD}^{+}{ }^{+} \mathrm{T}$ cells in male homosexual patients with $\mathrm{HIV}-1$ infection}

Li Li ${ }^{1}$, Ling-hang Wang ${ }^{1}$, Feng-ting Yu${ }^{1}$, Si-yuan Yang ${ }^{1}$, Shu-jing Song ${ }^{2}$, Yun-xia Tang ${ }^{1}$, Cheng-jie Ma ${ }^{*}$, Xing-wang $\mathrm{Li}^{*} 1$

${ }^{1}$ Clinical and Research Center of Infectious Disease, Beijing Ditan Hospital, Capital Medical University, Beijing 100015, China

${ }^{2}$ Clinical Laboratory of Beijing Ditan Hospital, Capital Medical University, Beijing 100015, China

*Correspondences:

Cheng-jie Ma, E-mail: mcj1126@ccmu.edu.com; Clinical and Research Center of Infectious Disease, Beijing Ditan Hospital, Capital Medical University, Beijing 100015, China.

Xing-wang Li: E-mail address: ditanlxw@163.com; Clinical and Research Center of Infectious Disease, Beijing Ditan Hospital, Capital Medical University, Beijing 100015, China.

Short title: T cell aging in male homosexual HIV subjects

Key Words: HIV, CD4 ${ }^{+} \mathrm{T}$ cell, Immune activation, Premature aging, Telomere

List of abbreviations: 
ART, antiretroviral therapy; PBMC, peripheral blood mononuclear cell; TN, naive T; TCM, central memory $\mathrm{T}$; TEM, effector memory $\mathrm{T}$; TemRA, terminally differentiated memory $\mathrm{T}$; HC, healthy controls; IQR, interquartile range; $\mathrm{SD}$, standard deviation; ANOVA, analysis of variance.

\section{Summary}

The average lifespan of HIV-infected subjects remains shorter compared to uninfected individuals. Immunosenescence may be responsible for this difference despite effective antiretroviral therapy (ART) with successful viral suppression. Here, we evaluated the effects of HIV and ART exposure on T cell aging in male homosexual HIV subjects. CD4 ${ }^{+} \mathrm{T}$ cell activation $\left(\mathrm{HLA}-\mathrm{DR}{ }^{+}\right)$and senescence $\left(\mathrm{CD} 57^{+}\right)$markers were analyzed by flow cytometry, and telomere length was quantified by real-time PCR. Specifically, we observed an increase in activation and senescence markers on total CD4 ${ }^{+} \mathrm{T}$ cell populations in HIV-infected subjects. We also observed a reduction in senescence markers on terminally differentiated memory $\mathrm{T}(\mathrm{TemRA})$ cells and activation markers on central memory T(TCM), effector memory T(TEM), and TemRA cells in ARTtreated HIV subjects. Furthermore, we also observed an extension of telomere length in memory CD4 ${ }^{+} \mathrm{T}$ cells, rather than naive CD4 ${ }^{+} \mathrm{T}$ cells, after viral control by ART. Our results indicate that HIV-infected patients exhibit a premature $\mathrm{T}$ cell aging phenotype with accelerated immune senescence. Partial recovery of immune senescence and differentiation aberrances is achieved in CD4 ${ }^{+} \mathrm{T}$ cells in HIV patients on ART. Overall, these results suggest that HIV infection, rather than ART exposure, influences the T cell aging process.

\section{Introduction}

Treatment of HIV-infected patients with combination antiretroviral therapy (ART) has led to a significant reduction in both morbidity and mortality. Therefore, HIV infection has transitioned from an acute, terminal illness to a chronic but manageable condition [1]. While this is undoubtedly a major success, several studies have demonstrated that HIV-infected individuals are at an increased risk of age-related non-AIDS morbidity and mortality compared with uninfected persons [2]. These observations have led to the proposal that HIVinfected individuals suffer from accelerated or premature aging [3]. However, the pathogenic mechanisms underlying this increased aging process remain poorly understood.

Growing evidence suggests that immune exhaustion and senescence caused by HIV infection is similar to that caused by age in uninfected elderly subjects [4-6]. During nature aging, a reduction in T cell renewal occurs together with a progressive enrichment of terminally differentiated $\mathrm{T}$ cells with shortened telomeres. It is thought that these changes are the consequence of immune activation and inflammation, which translates into a general decline of the immune system, which gradually leads to immunosenescence (aging of the immune system) [7]. Similarly, high levels of systemic immune activation and inflammation due to HIV infection promote the accelerated replicative senescence of $\mathrm{T}$ cells [8], which leads to an imbalance of $\mathrm{T}$ cell phenotype $[9,10]$. This results in the differentiation and accumulation of nonfunctional senescent $\mathrm{T}$ cells [6]. In addition, studies have shown that premature and accelerated $\mathrm{T}$ cell aging in HIV-infected patients can be caused by the adverse effects of antiretroviral drugs [11,12]. Hopefully, data from a recent clinical study convincingly suggest that the initiation of ART can lead to rapid improvements in several clinical outcomes $[13,14]$; however, the extent to which these immunological signatures were restored during ART has not been determined. Moreover, little is known about the combined impact of HIV infection and ART drugs on immunosenescence in HIV-infected individuals.

Although some reports have characterized distinct $\mathrm{T}$ cell subsets in HIV-infected subjects and compared their distribution with those in healthy subjects $[15,16]$, few data are available regarding the activation/senescent profile in relation to the immunosenescence features of distinct $\mathrm{T}$ cell subsets. In addition, no reports have provided a comprehensive assessment of telomere length of distinct $\mathrm{T}$ cell subsets, of which telomere shortening represents a key molecular marker of biological aging. In the current study, we provide a detailed characterization of biological aging in relation to immune senescence and activation markers of the different $\mathrm{CD} 4^{+} \mathrm{T}$ cell subsets in a cohort of male homosexual HIV-infected subjects, including ART-naive and ARTtreated subjects. 


\section{Materials and methods}

\section{Study subjects}

A total of 60 HIV-infected patients were recruited from the Outpatient Clinic of Beijing Ditan Hospital, Capital Medical University (China). A total of 30 patients were ART-naive at the time of enrolment (ARTnaive group). There were 30 patients who received ART for a minimum of one year prior to inclusion and had suppressed viral replication (ART-receiving group). For comparison, 28 age-matched HIV-negative healthy subjects were included as a control group (HC group). Both HIV-infected individuals and healthy controls were all homosexual males in this study. The exclusion criteria consisted of chronic diseases, neoplasms, immune inflammatory diseases, other non-HIV-related diseases, and metabolic complications. This study was approved by the local research ethics committee. Written informed consent was obtained from all participants.

\section{$\mathrm{CD}^{+} \mathrm{T}$ cell count and plasma HIV-1 viral load}

Fasting venous blood samples were collected. CD4 ${ }^{+} \mathrm{T}$ cell count (cells/L) and HIV-1 viral load (HIV-RNA, copies $/ \mathrm{mL}$ ) were determined in all $\mathrm{HIV}$-infected subjects. Absolute $\mathrm{CD} 4^{+} \mathrm{T}$ cell numbers were determined using an Epics XL-MCL flow cytometer (Beckman Coulter, Brea, CA, USA). Plasma HIV-1 RNA levels were determined using the COBAS Taqman HIV-1 test (Roche, Branchburg, New Jersey, USA) according to the manufacturer's instructions. The detection limit was 40 HIV RNA copies/mL.

\section{Flow Cytometry analysis}

All analyses were performed using fresh peripheral blood mononuclear cell (PBMC) samples, isolated by density centrifugation using Ficoll-Hypaque (Amersham Biosciences, Amersham, Buckinghamshire, United Kingdom) from $20 \mathrm{~mL}$ EDTA and venous blood. The following monoclonal antibodies were used for T cell immunophenotyping: CD4-APC-CY7, CD8-FITC, CD28-APC, CD45RA-PE-CY7, CCR7-PERCP-CY5.5, CD27-AmCyan HLA-DR-Pacific Blue, and CD57-PE (BD Biosciences, San Jose, CA). Combinations of CD45RA, CCR7, CD28, and CD27 are commonly employed to define four different T cell subsets [17]: 1) $\mathrm{CD}_{2} 8^{++} \mathrm{CD} 27^{++} \mathrm{CCR} 7{ }^{+} \mathrm{CD} 45 \mathrm{RA}^{+}$[naive $\mathrm{T}$ cells (TN)]; 2) $\mathrm{CD} 28^{+++} \mathrm{CD} 27^{++} \mathrm{CCR} 7^{+} \mathrm{CD} 45 \mathrm{RA}^{-}[$central memory T cells (TCM)]; 3) CD28+ ${ }^{+}$CD27 ${ }^{+}$-[effector memory T cells (TEM)]; and CD28- CD27-[ (terminally differentiated memory T cells (TemRA)]. Cellular activation in CD4 subsets was characterized by the expression of HLA-DR [18]. Senescent CD4 ${ }^{+}$T cells were characterized by CD57 expression [19]. Fluorescence was measured with a FACS Canto II (BD Biosciences, Breda, the Netherlands). A total of 100,000 events were collected in the lymphocyte gate using morphological parameters (forward and side-scatter). The raw data were analysed using a fluorescence activated cell sorter (FACS) Diva version 5 (BD Biosciences, Heidelberg, Germany) and FlowJo software (TreeStar Inc., Ashland, OR, USA).

\section{Isolation of naive and memory $T$ cells}

Lymphocyte subpopulations were isolated from fresh PBMCs using magnetic beads (AutoMACS; Miltenyi Biotec) coated with monoclonal Abs to CD4, CD8, CD45RA, and CD45RO, in accordance with the manufactures' instructions. The isolated subpopulations were stained with immunofluorescently-labelled monoclonal Abs to CD4, CD8, CD45RA, and CD45RO. Isolated $\mathrm{CD}^{+} \mathrm{CD} 45 \mathrm{RA}^{+}$cells (naive $\mathrm{T}$ cells) and $\mathrm{CD}^{+}{ }^{+} \mathrm{CD} 45 \mathrm{RO}^{+}$cells (memory $\mathrm{T}$ cells) from both the patients and controls were frozen in 90\% FCS/10\% DMSO in the vapor phase oliquid nitrogen until future use. Samples for the isolation of naive and memory T cells were available for $24 \mathrm{HC}, 21$ ART naive, and 18 ART.

\section{DNA extraction and measurement of telomere length by quantitative real-time PCR}

After isolating naive and memory $\mathrm{T}$ cells, the DNA was extracted using a QIAamp RNA Mini Kit (Qiagen) according to the manufacturers' instructions. The concentration and purity of the DNA was quantified using a Nanodrop Spectrophotometer (ThermoFisher Scientific, Waltham, Massachusetts, USA). The telomere length was determined by quantitative real-time PCR adapted from Cawthon [20] . The relative telomere length was measured as the ratio of standard DNA quantities for telomere template $(\mathrm{T})$ 
over single copy gene, 36B4 (S). The telomere and 36B4 gene primers were as follows: (Tel-forward,5'CGGTTTGTTTGGGTTTGGGTTTGGGTTTGGGTTTGGGTT-3'; Tel reverse, 5'-GGCTTGCCTTACCCTTACCCTTAC 3') for Telomere PCR and (36B4-forward, 5'-CAGCAAGTGGGAAGGTGTAATCC-3'; 36B4-reverse, 5'CCCATTCTATCATCAACGGGTACAA-3') for the 36B4 PCR. All samples were blindly and consecutively run in triplicate together with reference samples. The intra-assay coefficient of variation was $5 \%$.

\section{Statistical analysis}

All continuous variables were expressed as the median with an interquartile range(IQR) or mean with standard deviation(SD), as appropriate. All groups were tested for the normal distribution with a KolmogorovSmirnov test. For the normally distributed data, a one-way analysis of variance (ANOVA) was used to analyze more than two groups. Data that were not normally distributed were tested by a one-way KruskalWallis test for more than two groups. All statistical analysis was carried out using Graphpad Prism version 5 (GraphPad, La Jolla, CA, USA) and SPSS 22.0 (College Station, TX, USA) software. In the graphs, the $\mathrm{p}$ values are indicated as follows: ${ }^{*}<0.05 ;{ }^{* *}<0.01 ; * * *<0.001$.

\section{Results}

\section{Characteristics of study population}

A total of 30 untreated (ART naive) HIV-infected subjects (28 years old; IQR: 26 34 years) and 30 ARTtreated HIV subjects (30.5 years old; IQR: $26^{\sim} 36.5$ years) as well as 28 healthy subjects ( 28 years old; IQR: $26 \sim 34$ years) were included in this study. As shown in Table 1, HIV-infected subjects and healthy subjects did not significantly differ in age. $\mathrm{CD} 4^{+} \mathrm{T}$ cell counts were only available for HIV-infected subjects. The median $\mathrm{CD} 4^{+} \mathrm{T}$ cell counts were significantly lower in the ART-naive HIV-infected subjects with a detectable viral load $(p<0.0001)$. The ART-treated HIV subjects had undergone triple therapy by lamivudine (3TC) + tenofovir (TDF) + efavirenz (EFV) for a mean duration of $13.0(12.0,22.0)$ months and had an undetectable plasma viral load $(<40$ copies $/ \mathrm{mL})$.

\section{Phenotypic T cell alterations occur early in male homosexual HIV-1 infection}

HIV infection is characterized by a disturbance in $\mathrm{T}$ cell homeostasis, which results in an alteration to $\mathrm{T}$ cell subsets. To investigate the impact of HIV infection on $\mathrm{CD} 4^{+} \mathrm{T}$ cell populations, we performed a cross-sectional analysis of $\mathrm{CD} 4^{+} \mathrm{T}$ cell populations in male homosexual HIV patients compared to healthy controls. Patients with chronic HIV infection were stratified into ART-receiving and ART-naive patients. The phenotypic characteristics of the $\mathrm{CD} 4^{+} \mathrm{T}$ cell subsets are presented in Table 2 . Within $\mathrm{CD} 4^{+} \mathrm{T}$ cells, the percentage of TN was lower in the HIV-infected patients ( $\left.\mathrm{n}=30,34.10\left[12.38^{\sim} 47.73\right] \%\right)$ than in the control group ( $\mathrm{n}=28,43.26$ [35.20 52.08] \%, $p=0.0209$ ) (Fig.1a). For the TCM, although the difference was not statistically significant, it tended to be more expanded in HIV-infected patients ( $\mathrm{n}=30,32.95$ [28.13 39.68] $\%)$ than in the control group $\left(\mathrm{n}=28,32.10\left[27.67^{\sim} 36.68\right] \%\right)$ (Fig.1b). Interestingly, statistically significant frequencies of TEM and TemRA were observed in two groups $(p<0.0001$ and $p=0.0061$, respectively) (Fig.1c and d). To study the effect of ART on $\mathrm{CD}^{+} \mathrm{T}$ cell subsets, the differences between ART-naive HIVinfected patients and ART-receiving patients were further analyzed. The percentage of TN was expanded to a greater extent in ART-receiving patients $(\mathrm{n}=30,43.35$ [38.38 48.13] \%, $p=0.0314)$ when compared to ART-naive patients $\left(\mathrm{n}=30,34.10\left[12.38^{\sim} 47.73\right] \%, p=0.0314\right)$ (Fig.1a). Similarly, there was no difference in the frequencies of TCM between the two groups. While the percentages of TEM and TemRA were significantly reduced in the ART-receiving patients $\left(\mathrm{n}=30,13.43\left[9.40^{\sim} 19.81\right] \%\right.$ and $0.4\left[0.175^{\sim} 0.625\right] \%$, respectively) in comparison to ART-naive patients $(\mathrm{n}=30,18.50$ [11.93 23.85] \% and 1.000 [0.3 1.783] \%, $p=0.0157$ and $p=0.0274$, respectively) (Fig. $1 \mathrm{c}$ and $\mathrm{d}$ ).

Increased frequencies of $\mathrm{CD}^{+} 7^{+}$and $\mathrm{HLA}-\mathrm{DR}{ }^{+}$cell populations in $\mathrm{CD}^{+} \mathrm{T}$ cell subsets in male homosexual HIV-1 infection

We next analyzed alterations in the expression of replicative senescence and activation markers in the different $\mathrm{CD} 4^{+} \mathrm{T}$ cell subsets. The results are presented in Table 2. For TN, the frequencies of CD $57^{+}$cells were elevated within HIV-infected patients $\left(\mathrm{n}=30,1.425\left[0.905^{\sim} 3.968\right] \%\right)$ compared to healthy controls $(\mathrm{n}=$ 
28, $0.95\left[0.5425^{\sim} 1.728\right] \%, p=0.0297$ ) (Fig. 2a). Similar expansion was observed in the TCM, TEM, and TemRA subsets ( $p=0.0041,0.0032$, and $p<0.0001$, respectively) (Fig. 2c, e, and g). When we measured the level of HLA-DR expression, we observed a similar expression pattern compared to CD57 regarding elevated HLA-DR expression on all $\mathrm{CD} 4^{+} \mathrm{T}$ cell subsets (Fig. 2b, d, f, h). Thus, a higher percentage of CD57 and HLA-DR expression on CD4 ${ }^{+} \mathrm{T}$ cell subsets was observed in HIV-infected patients compared to healthy controls. This suggests accelerated immune senescence in HIV-infected patients. On the other hand, the effects of ART on CD4 ${ }^{+} \mathrm{T}$ cell surface senescence and activation markers were also analyzed. When the frequencies of $\mathrm{CD} 57^{+}$cells of the different $\mathrm{CD} 4^{+} \mathrm{T}$ cell subsets were analyzed, we only observed a significant reduction on TemRA cells in ART-receiving patients $(\mathrm{n}=30,61.85$ [49.4 100]\%) compared to that of ART-naive subjects $\left(\mathrm{n}=30,76.80\left[66.70^{\sim} 100\right] \%, p=0.0358\right)$ (Fig. 2g), but not on TN, TCM, or TEM. Similar effects were observed for the frequencies of HLA-DR ${ }^{+}$cells of different CD4 ${ }^{+} \mathrm{T}$ cell subsets, showing a significant decrease in frequencies of HLR-DR ${ }^{+}$cells on TCM, TEM, and TemRA compared to ART-naive patients ( $p=0.0006,0.0269$, and 0.0019, respectively) (Fig. 2d, f, and h); however, there was no statistically significant difference in TN.

Collectively, our data demonstrate increased frequencies of $\mathrm{CD}^{+} 7^{+}$and HLA-DR ${ }^{+}$cell populations in most $\mathrm{CD}^{+} \mathrm{T}$ cell subsets in chronically HIV-infected subjects, which can be partially reversed following ART.

\section{Telomere length of memory $\mathrm{T}$ cells is shorter in male homosexual HIV-1 infection}

Telomeres are specialized structures comprised of tandem repeats of TTAGGG located at the end of chromosomes and are essential for chromosomal stability. An assessment of telomere length is used to evaluate cellular replication history or senescent status. Moreover, immune activation and microbial translocation are thought to drive telomere shortening in HIV infection [21]. To investigate the impact of chronic HIV infection and ART on the telomere length of distinct $\mathrm{T}$ cell subsets, we further determined the telomere length of both naive and memory $\mathrm{T}$ cells isolated from HIV patients. For naive $\mathrm{T}$ cells, there was no significant alteration in telomere length between healthy controls and HIV-infected patients, nor between the ART-naive patients and ART-receiving patients. For memory T cells, however, ART-naive patients had a significantly decreased telomere length compared to that of healthy controls and ART-receiving patients, at $2.96 \pm 2.40,7.82 \pm$ 3.58 , and $6.27 \pm 3.23$, respectively $(p<0.0001$ and $p=0.0058)$. However, the differences in telomere length between the healthy controls and ART-receiving patients were not statistically significant.

\section{Discussion}

Chronic HIV infection can drive premature T cell aging, even in the setting of ART with complete viral suppression. In this study, we demonstrate that male homosexual HIV subjects exhibit premature biological aging with accelerated immune senescence, which affects $\mathrm{CD} 4^{+} \mathrm{T}$ cell subsets that have partial recovery by ART.

In the context of HIV infection, disruption of normal $\mathrm{T}$ cell homeostasis has been observed when a massive and continuous depletion of $\mathrm{CD} 4^{+} \mathrm{T}$ cells occurs. Compared with healthy age-matched subjects, a lower [15] or similar [16] $\mathrm{CD}^{+}$naive $\mathrm{T}$ cell frequency has been reported. As expected, the results of this study show a lower $\mathrm{CD}^{+}$naive T cell frequency in HIV-infected subjects compared with healthy subjects. This may be partially explained by the capacity of the thymus to produce new $\mathrm{T}$ cells or thymic output, which is significantly decreased during the course of HIV infection [22-24]. Overall, the frequency of naive T cells represents a good marker of immunological age in humans, and its progressive decrease in HIV-infected adults is typically directly associated with HIV disease progression [25]. A significant increase in memory cell populations is observed when compared to the uninfected controls in our study, especially in TEM, which is consistent with a previous report [26]. Moreover, ART treatment result in a partial restoration of the pool of naive as well as memory $\mathrm{T}$ cells, which show an increase in naive $\mathrm{T}$ cells and a reduction of TEM and TemRA cell populations, although a return to normal levels has seldom been observed. Overall, our data support a notion in which active HIV replication drives the production of a senescent phenotype, resulting in a decline in $\mathrm{T}$ cell competence.

CD57 has been described as a marker of replicative senescence in T cells, and is associated with telomere 
shortening following numerous cell divisions [19]. Interestingly, this marker, along with the marker of T cell activation (HLA-DR), is recently used to assess immune activation and senescence, which is correlated with the clinical status of HIV-infected patients and is closely associated with the increase in non-AIDS-related morbidities (e.g., cardiovascular disease, chronic liver disease, kidney disease, osteoporosis, neurocognitive disease, and cancer) in HIV-suppressed patients with age $<60$ years old [27]. In our study, we observe higher CD57 and HLA-DR expression in the expanded CD4 ${ }^{+}$memory $\mathrm{T}$ cell populations in HIV-infected subjects, with the exception of naive $\mathrm{T}$ cells. However, the decreased levels of specific $\mathrm{CD} 4^{+} \mathrm{T}$ cell subsets may reflect the effect of $\mathrm{ART}$ that can reduce $\mathrm{CD} 4^{+} \mathrm{T}$ cell activation [28]. Moreover, immune senescence is greater in HIV-infected subjects with detectable HIV viremia. This indicates that active HIV replication leads a senescent phenotype, which demands for the early control of HIV replication and chronic immune activation. Of note, despite effective suppression of viral replication, ART fails to re-establish immunological homeostasis in the treated patients, which is indicative of a fully exhausted phenotype.

Telomeres play an important role in controlling both cultured cell senescence in vitro and the aging process in vivo[29]. As a classical marker of cell senescence, the shortening of telomere length is often used as an indicator of individual biological age [30]. Some reports [31,32] have analyzed telomere length, which is usually measured in total PBMCs or total T cell subsets (CD4 or CD8), demonstrating that telomere length was shorter in HIV-infected individuals compared to age-matched seronegative controls. This suggests that there is accelerated immunological aging with HIV infection. During the course of HIV infection, the distribution of CD4 cell subsets is known changed, and telomere length is also varied in distinct cell subsets according to the stage of differentiation (e.g., naive and memory cells). To date, there is limited data available regarding the telomere length of distinct $\mathrm{T}$ cell subsets. In our study, no significant differences are observed in telomere length of naive T cells between HIV-infected subjects and healthy controls. Consistent with a previous report [33], we also find that ART had no obvious effect on the telomere length of naive T cells, which indicates that either HIV infection or exposure to ART had little influence on naive T cells. However, ART-naive subjects had significantly shorter telomeres in memory $\mathrm{T}$ cells compared to healthy controls and those HIV subjects on ART, indicating that HIV infection, rather than exposure to ART, affects the aging process. However, it has been reported that ART may inhibit telomerase activity, leading to differences in telomere lengths between the ART-naive and ART-treated groups. Previous studies have inconsistent findings in such an association [34-36]. Therefore, a further comprehensive analysis of telomere length in different $\mathrm{T}$ cell subsets in accordance with the stage of $\mathrm{T}$ cell differentiation, the effect of ART on telomere length, and potential mechanisms are required in future.

In this study, we analyzed the immunosenescence of $\mathrm{CD} 4^{+} \mathrm{T}$ cell subsets in male homosexual patients with HIV-1 infection. Unfortunately, our current cohort is too small to stratify CD4 ${ }^{+} \mathrm{T}$ cells in patients receiving antiretroviral therapy for their $\mathrm{CD} 4^{+} \mathrm{T}$ cell nadir.

In conclusion, male homosexual subjects with HIV-1 infection exhibit premature aging with accelerated immune senescence, which affects the $\mathrm{CD} 4^{+} \mathrm{T}$ cell subsets. The higher expression of senescent markers on different $\mathrm{CD}^{+} \mathrm{T}$ cell subsets in ART-naive subjects, compared with ART-receiving subjects or healthy controls, suggests that HIV infection, rather than ART exposure, influences the aging process. Our data support the importance of early HIV control to avoid premature immune senescence to reduce the risk of age-related diseases, including malignancies. Our findings can also be interpreted as a correlation between $\mathrm{CD}^{+} \mathrm{T}$ cell senescence and premature aging of the immune system, which can continue to be observed despite successful viral suppression.

\section{Acknowledgements}

We thank the study participants and the staff that collected the blood samples at the Clinical and Research Center of Infectious Disease, Beijing Ditan Hospital. This work was funded by National Science and Technology Major Project during the '13th Five-year' Plan Period (No.2017ZX10205502).

Author contributions

Li Li, Cheng-jie Ma and Xing-wang Li conceived the study and designed the protocol. Li Li, Feng-ting Yu 
and Shu-jing Song performed the experiments. Li Li, Ling-hang Wang and Si-yuan Yang analyzed obtained data. Li Li and Yun-xia Tang collected samples. Cheng-jie Ma and Xing-wang Li supervised the project. $\mathrm{Li} \mathrm{Li}$ wrote the manuscript. All authors have reviewed the final version of the manuscript and approved its submission for publishing.

\section{Conflict of interest}

The authors declare no commercial or financial conflict of interest.

\section{References}

1 Bhatia R, Ryscavage P, Taiwo B. Accelerated aging and human immunodeficiency virus infection: Emerging challenges of growing older in the era of successful antiretroviral therapy. J Neurovirol 2012;18 :247-55.

2 Deeks SG. Immune dysfunction, inflammation, and accelerated aging in patients on antiretroviral therapy. Top HIV Med 2009;17 :118-23.

3 Capeau J. Premature Aging and Premature Age-Related Comorbidities in HIV-Infected Patients: Facts and Hypotheses. Clin Infect Dis 2011;53 :1127-9.

4 Deeks SG. HIV infection, inflammation, immunosenescence, and aging. Annu Rev Med 2011; 62 :141-55.

5 Molina-Pinelo S, Vallejo A, Díaz L, Soriano-Sarabia N, Ferrando-Martínez S, Resino S, Muñoz-Fernández MA, Leal M. Premature immunosenescence in HIV-infected patients on highly active antiretroviral therapy with low-level CD4 T cell repopulation. J Antimicrob Chemother 2009; 64 :579-88.

6 Desai S, Landay A. Early Immune senescence in HIV disease. Curr HIV/AIDS Rep 2010; 7 :4-10.

7 Bischof J, Gärtner F, Zeiser K et al. Immune cells and immunosenescence. Folia Biol (Praha) 2019; 65 $: 53-63$.

8 Appay V, Sauce D. Immune activation and inflammation in HIV-1 infection: causes and consequences. J Pathol 2010; $214: 231-41$.

9 Kelley CF, Kitchen CMR, Hunt PW. et al. Incomplete peripheral CD4+ cell count restoration in HIVinfected patients receiving long-term antiretroviral treatment. Clin Infect Dis 2009; 48 :787-94.

10 Douek DC, Mcfarland RD, Keiser PH. et al. Changes in thymic function with age and during the treatment of HIV infection. Nature 1998; $396: 690-5$.

11 Franzese O, Barbaccia ML, Bonmassar E, Graziani G. Beneficial and Detrimental Effects of Antiretroviral Therapy on HIV-Associated Immunosenescence. Chemotherapy (Los Angel) 2018; 63:64-75.

12 Cohen J, Torres C. HIV-associated cellular senescence: a contributor to accelerated aging. Ageing Res Rev $2017 ; 36: 117-24$.

13 Eberhard JM, Ahmad F, Hong HS, Bhatnagar N, Keudel P, Schulze Zur Wiesch J, Schmidt RE, MeyerOlson D. Partial recovery of senescence and differentiation disturbances in CD8(+) T cell effector-memory cells in HIV-1 infection after initiation of ART. Clin Exp Immunol 2016;186 :227-38.

14 Behrens NE, Wertheimer A, Klotz SA, Ahmad N. Reduction in terminally differentiated T cells in virologically controlled HIV-infected aging patients on long-term antiretroviral therapy. PLoS One 2018;13 :e0199101-0199101.

15 Ananworanich J, Apornpong T, Kosalaraksa P. et al.Characteristics of lymphocyte subsets in HIVinfected, long-term non-progressor, and healthy Asian children through 12 years of age. J Allergy Clin Immunol 2010; 126 :1294-301.e10.

16 Ssewanyana I, Baker CA, Ruel T. et al. The Distribution and Immune Profile of T Cell Subsets in HIV-Infected Children from Uganda. AIDS Res Hum Retroviruses 2008; 25 :65-71. 
17 McLane LM, Banerjee PP, Cosma GL, Makedonas G, Wherry EJ, Orange JS, Betts MR. Differential localization of T-bet and Eomes in CD8 T-cell memory populations. J Immunol 2013; 190 :3207-15.

18 De MM, Rossi ME, Azzari C, Gelli MG, Galli L, Vierucci A. Different meaning of CD38 molecule expression on $\mathrm{CD} 4+$ and CD8+ cells of children perinatally infected with human immunodeficiency virus type 1 infection surviving longer than five years. Pediatr Res 1998; 43 :752-8.

19 Brenchley JM, Karandikar NJ, Betts MR. et al. Expression of CD57 defines replicative senescence and antigen-induced apoptotic death of CD8+ T cells. Blood 2003; 101 :2711-20.

20 Cawthon RM. Telomere length measurement by a novel monochrome multiplex quantitative PCR method. Nucleic Acids Res 2009;37 :e21.

21 Deeks SG, Verdin E, Mccune JM. Immunosenescence and HIV. Curr Opin Immunol 2012; 24 :501-6.

22 Dion, MarieLise, Poulin. et al. HIV Infection Rapidly Induces and Maintains a Substantial Suppression of Thymocyte Proliferation. Immunity 2004; 21 :757-68.

23 Fabre-Mersseman V, Dutrieux J, Louise A. et al. CD4+ recent thymic emigrants are infected by HIV in vivo, implication for pathogenesis. AIDS 2011; $25: 1153-62$.

24 Lelièvre JD, Melica G, Itti E. et al. Initiation of c-ART in HIV-1 infected patients is associated with a decrease of the metabolic activity of the thymus evaluated using FDG-PET/computed tomography. J Acquir Immune Defic Syndr 2012; 61 :56-63.

25 Roederer M, Dubs JG, Anderson MT, Raju PA, Herzenberg LA, Herzenberg LA. CD8 naive T cell counts decrease progressively in HIV-infected adults. J Clin Invest 1995; 95 :2061-6.

26 Méndez-Lagares G, Díaz L, Correa-Rocha R. et al. Specific patterns of CD4-associated immunosenescence in vertically HIV-infected subjects. Clin Microbiol Infect 2013; 19 :558-65.

27 Duffau P, Wittkop L, Lazaro E. et al. Association of immune-activation and senescence markers with non-AIDS-defining comorbidities in HIV-suppressed patients. AIDS 2015;29 :2099-108.

28 Resino S, Navarro J, Bellón JM, Gurbindo D, León JA, Muñoz-Fernández MA. Naïve and memory CD4+ $\mathrm{T}$ cells and T cell activation markers in HIV-1 infected children on HAART. Clin Exp Immunol 2010; 125 :266-73.

29 Shay JW, Wright WE. Hallmarks of telomeres in ageing research. J Pathol 2010; 211 :114-23.

30 Appay V, Sauce D. Assessing immune aging in HIV-infected patients. Virulence 2017; 8 :529-38.

31 Wolthers KC, Wisman G, Otto SA. et al. T cell telomere length in HIV-1 infection: No evidence for increased CD4+ T cell turnover. Science 1996;274:1543-7.

32 Ketty G, Antoni NJ, Marisa Z. et al. Premature aging and immune senescence in HIV-infected children. AIDS 2016;30:1363-73.

33 Tucker V, Jenkins J, Gilmour J, Savoie H, Easterbrook P, Gotch F, Browning MJ. T-cell telomere length maintained in HIV-infected long-term survivors. HIV Med 2010; 1 :116-22.

34 Leeansyah E, Cameron PU, Solomon A. et al. Inhibition of telomerase activity by human immunodeficiency virus (HIV) nucleos(t)ide reverse transcriptase inhibitors: a potential factor contributing to HIV-associated accelerated aging. J Infect Dis 2013;207 :1157-65.

35 Ballon G, Ometto L, Righetti E, Cattelan AM, Masiero S, Zanchetta M, Chieco-Bianchi L, De Rossi A. Human immunodeficiency virus type 1 modulates telomerase activity in peripheral blood lymphocytes. J Infect Dis 2001; 183 :417-24. 
36 Wolthers KC, Otto SA, Wisman GB, Fleury S, Reiss P, ten Kate RW, van der Zee AG, Miedema F. Normal T-cell telomerase activity and upregulation in human immunodeficiency virus-1 infection. Blood $1999 ; 93: 1011-9$.

\section{Figure legend}

Figure 1. Impact of HIV infection and ART on frequencies of CD4+ T cell subsets. Flow cytometry analyses of PBMCs in HIV-infected and healthy subjects.(A) Gating strategy to identify CD4+ T cell subsets(TN, TCM, TEM, TemRA).(B) The frequencies of CD4+ T cell subsets in HIV-infected and healthy subjects, including TN(a), TCM(b), TEM(c), TemRA(d). Measurements were performed on samples from 30 ART naive patients, 30 ART patients and 28 healthy subjects. About 5 PBMC samples were analyzed fresh within a working day and a total of 18 independent experiments were performed. Median [IQR] values are indicated. Significant differences were determined in a non-parametric Kruskal-Wallis test: ${ }^{*} p<0.05$, ${ }^{* *} p<0.01,{ }^{* * *} p<0.001$.

Figure 2. Impact of HIV infection and ART on replicative senescence(CD57 ${ }^{+}$)and activation $\left(\right.$ HLADR $^{+}$) expression within CD4 T-cell subsets (see Fig.1) .

Flow cytometry analyses of PBMCs in HIV-infected and healthy subjects. (A)Left: Representative dot plots showing the proportion of CD4 subsets-expressing CD $57^{+} \mathrm{T}$ cells. Right: Representative dot plots showing the proportion of CD4 subsets-expressing HLADR ${ }^{+} \mathrm{T}$ cells.(B)Percentage of CD4 subsets-expressing $\mathrm{CD}^{5} 7^{+}$and HLADR $^{+} \mathrm{T}$ cells in HIV-infected and healthy subjects: including TN(a-b), TCM(c-d), TEM(e-f), TemRA(gh). Measurements were performed on samples from 30 ART naive patients, 30 ART patients and 28 healthy subjects. About 5 PBMC samples were analyzed fresh within a working day and a total of 18 independent experiments were performed. Median $[\mathrm{IQR}]$ values are indicated. Significant differences were determined in a non-parametric Kruskal-Wallis test: ${ }^{*} p<0.05,{ }^{* *} p<0.01,{ }^{* * *} p<0.001$.

Figure 3. Impact of HIV infection and ART on telomere length of naive and memory T cells in HIV-infected and healthy subjects .

Telomere length values of naive $\mathrm{T}$ cells(Left) and memory $\mathrm{T}$ cells(Right)were assessed. Measurements were performed on samples from 21 ART naive patients, 18 ART patients and 24 healthy subjects. Data are shown as mean $\pm \mathrm{SD}$ and are pooled from four independent experiments and the number of samples in each experiment is $24,16,16,7$, respectively. ${ }^{*} p<0.05,{ }^{* *} p<0.01$, ${ }^{* * *} p<0.001$; one-way analysis of variance .

\section{Hosted file}

table.docx available at https ://authorea.com/users/307000/articles/438002-immunosenescence-ofcd4-t-cells-in-male-homosexual-patients-with-hiv-1-infection 
A

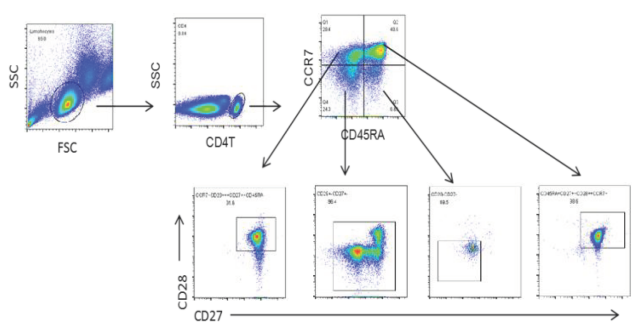

B

(a)

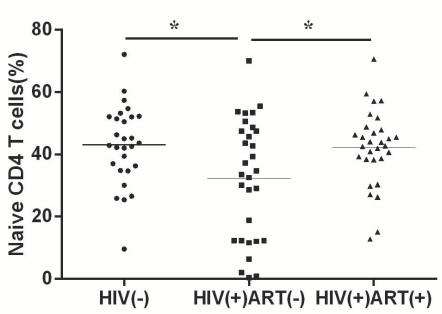

(c)

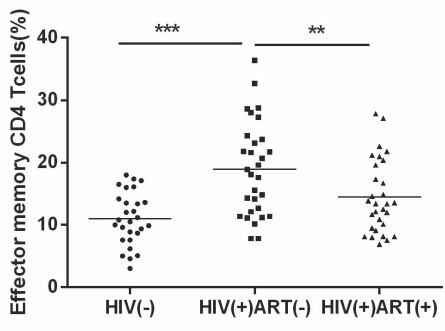

(b)

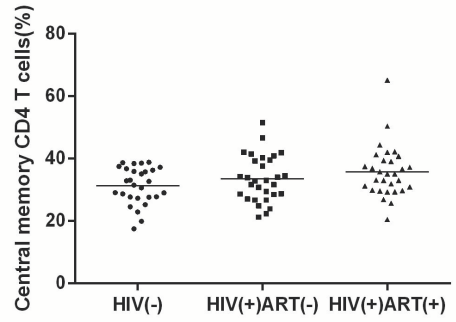

(d)

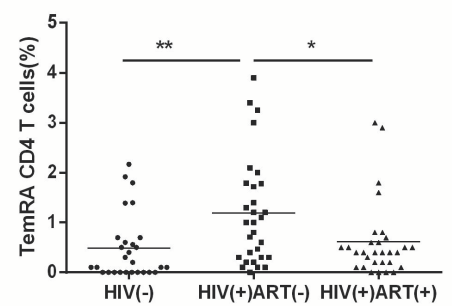

Figure 1. Impact of HIV infection and ART on frequencies of $\mathrm{CD}^{+} \mathrm{T}$ cell subsets. Flow cytometry analyses of PBMCs in HIV-infected and healthy subjects.(A) Gating strategy to identify $\mathrm{CD}^{+} \mathrm{T}$ cell subsets(TN, TCM, TEM, TemRA).(B) The frequencies of $\mathrm{CD}^{+}{ }^{+} \mathrm{T}$ cell subsets in HIV-infected and healthy subjects, including TN(a), TCM(b), TEM(c), TemRA(d). Measurements were performed on samples from 30 ART naive patients, 30 ART patients and 28 healthy subjects. About 5 PBMC samples were analyzed fresh within a working day and a total of 18 independent experiments were performed. Median [IQR] values are indicated. Significant differences were determined in a non-parametric Kruskal-Wallis test: ${ }^{*} p<0.05,{ }^{* *} p<0.01,{ }^{* * *} p<0.001$ 
A

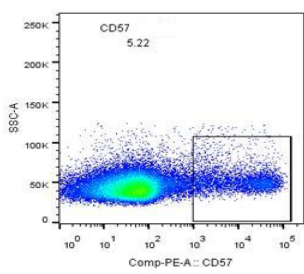

B

(a)

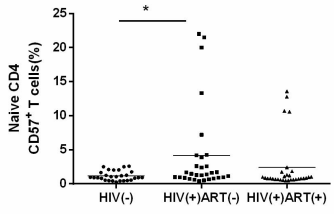

(c)

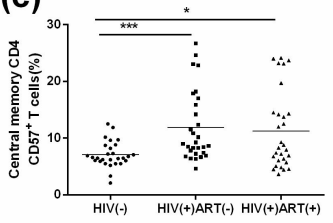

(e)

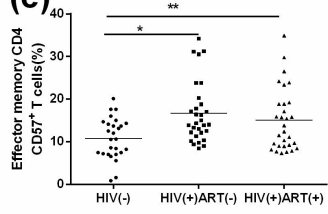

(g)

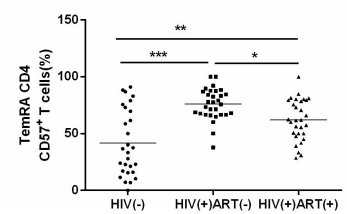

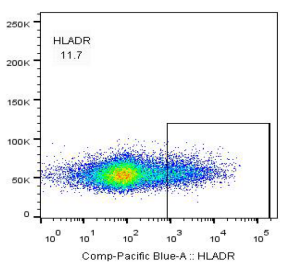

(b)

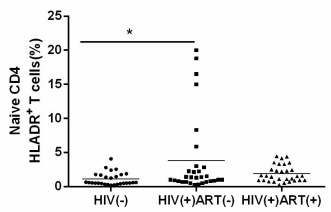

(d)

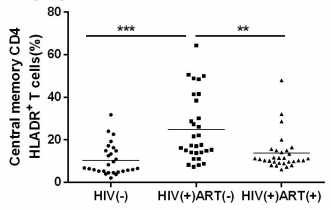

\section{(f)}

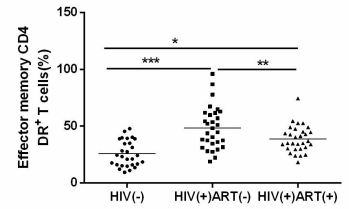

(h)

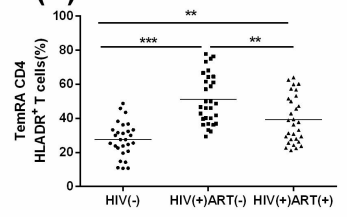

Figure 2. Impact of HIV infection and ART on replicative senescence $\left(\mathrm{CD} 57^{+}\right)$and activation $\left(\mathrm{HLADR}^{+}\right)$ expression within CD4 T-cell subsets (see Fig. 1). Flow cytometry analyses of PBMCs in HIV-infected and healthy subjects. (A)Left: Representative dot plots showing the proportion of CD4 subsets-expressing $\mathrm{CD}^{+} 7^{+} \mathrm{T}$ cells. Right: Representative dot plots showing the proportion of CD4 subsets-expressing $\mathrm{HLADR}^{+} \mathrm{T}$ cells.(B)Percentage of CD4 subsets-expressing $\mathrm{CD} 57^{+}$and $\mathrm{HLADR}^{+} \mathrm{T}$ cells in $\mathrm{HIV}$-infected and healthy subjects: including TN(a-b), TCM(c-d), TEM(e-f), TemRA(g-h). Measurements were performed on samples from 30 ART naive patients, 30 ART patients and 28 healthy subjects. About 5 PBMC samples were analyzed fresh within a working day and a total of 18 independent experiments were performed. Median $[\mathrm{IQR}]$ values are indicated. Significant differences were determined in a non-parametric Kruskal-Wallis test: $* p<0.05, * * p<0.01, * * * p<0.001$ 

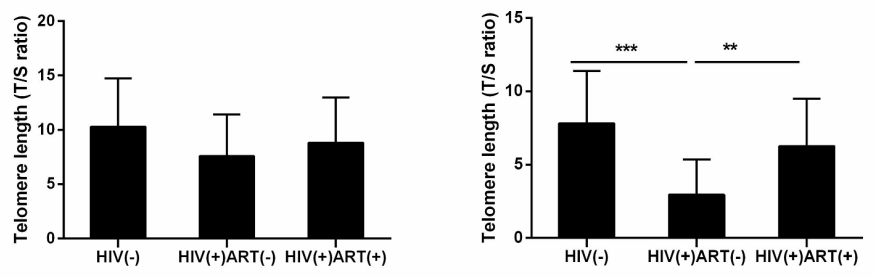

Figure 3. Impact of HIV infection and ART on telomere length of naive and memory T cells in HIV-infected and healthy subjects. Telomere length values of naive T cells(Left) and memory T cells(Right)were assessed. Measurements were performed on samples from 21 ART naive patients, 18 ART patients and 24 healthy subjects. Data are shown as mean \pm SD and are pooled from four independent experiments and the number of samples in each experiment is $24,16,16,7$, respectively. ${ }^{*} p<0.05,{ }^{*} p<0.01, * * * p<0.001$; one-way analysis of variance . 